\title{
The effects of music intervention on burn patients during treatment procedures: a systematic review and meta-analysis of randomized controlled trials
}

\author{
Jinyi Li ${ }^{1}$, Liang Zhou ${ }^{2}$ and Yungui Wang ${ }^{3 *}$
}

\begin{abstract}
Background: The treatment of burn patients is very challenging because burn injuries are one of the most severe traumas that can be experienced. The effect of music therapy on burn patients has been widely reported, but the results have been inconsistent. Thus, we performed a systematic review and meta-analysis of randomized controlled trials in burn patients to determine the effect of music during treatments.

Methods: We searched a variety of electronic databases, including MEDLINE (via PubMed), EMBASE, Cochrane Library, Psychinfo, VIP Database for Chinese Technical Periodicals (VIP) and China National Knowledge Infrastructure (CNKI) for relevant trials on the basis of predetermined eligibility criteria. from their first available date through February 2016. Our search focused on two key concepts: music interventions (including music, music therapy and music medicine) and physical activity outcomes (including pain, anxiety, burn characteristics, dressing changes, wound care, debridement and rehabilitation). Two reviewers independently screened records and extracted data from all eligible studies. Statistical heterogeneity was determined using Q-test and the $l^{2}$ statistic. The endpoints included standardized mean differences (SMDs) and 95\% confidence intervals (Cls). Publication bias was tested by Begg's funnel plot and Egger's test.

Results: A total of 17 studies met the inclusion criteria, for a total of 804 patients. A statistically significant difference in pain relief was demonstrated between music and non-music interventions (SMD $=-1.26,95 \% \mathrm{Cl}[-1.83,-0.68]$ ), indicating that music intervention has a positive effect on pain alleviation for burn patients. The results indicated that music interventions markedly reduced anxiety in individuals compared to non-music interventions (SMD $=-1.22,95 \% \mathrm{Cl}$ $[-1.75,-0.69])$. Correspondingly, heart rate decreases were found after treatments that included music interventions $(\mathrm{SMD}=-0.60,95 \% \mathrm{Cl}[-0.84,-0.36])$.

Conclusion: In summary, a positive correlation was found between treatments including music interventions and pain alleviation, anxiety relief, and heart rate reduction in burn patients. However, additional high-quality studies with carefully considered music interventions for burn patients are still needed.
\end{abstract}

Keywords: Music intervention, Burn patients, Pain, Anxiety, Meta-analysis, Systematic review

\footnotetext{
* Correspondence: c_q2014@163.com

${ }^{3}$ The Third Military Medical University, Chongaing 400038, China

Full list of author information is available at the end of the article
} 


\section{Background}

The treatment of burn patients is very challenging because burn injuries are one of the most severe traumas that can be experienced. As medical technology has advanced, the majority of burn patients are now being successfully resuscitated and typically undergo early escharotomy, skin transplantation, and antibiotic administration in addition to receiving nutritional support, which together dramatically decrease their mortality rate [1]. However, burn patients must still experience many painful procedures, including skin grafting, escharectomy, debridement, dressing changes and physical rehabilitation. Burn patients usually face a series of physiological and psychological problems during treatment. Pain is a major problem and occurs during all stages of treatment. The adequate management of pain may make recovery more tolerable and affect morbidity by means of prevention of elevated metabolism, thereby reducing the chance of malnutrition and deterioration of the immune system [2]. And the researchers question the safety of analgaesics and anxiolytics in patients with major burns because of their requirement for massive fluid resuscitation has the potential for contributing to hemodynamic instability [3]. Moreover, the use of sedation and analgesia must be limited in pediatric burn patients. There is a very close relationship between anxiety and pain [4], and anxiety is the most common emotional issue faced by burn patients, as reported in early studies $[5,6]$. As such, the treatment of burn patients must incorporate a holistic view of pain and anxiety. Effectively adjusting treatment parameters to manage pain and anxiety is necessary for burn patients throughout treatment.

The use of music interventions in the clinic has a long history. These interventions have typically been used during treatment and rehabilitation. To date, many studies have reported the use of music as an intervention during dental procedures, surgery, chemotherapy, and injections [7-12]. Music interventions have also been used to manage pain and anxiety in patients during medical procedures for many years. A study reported by Bradt showed the effects of music interventions on preoperative anxiety in surgical patients [8]. Furthermore, studies conducted by Chlan et al. have shown that the use of music interventions can reduce anxiety in ICU patients on mechanical ventilation $[13,14]$. Other authors have also demonstrated anxiety reduction in mechanically ventilated ICU patients through music interventions [15-17]. Wang et al. [18] has indicated that the use of music interventions can significantly improve pain score, anxiety, heart rate, arterial pressure, and satisfaction score for patients undergoing a variety of endoscopic procedures. Notably, Hole et al. [19] demonstrated that music can help alleviate postoperative pain, anxiety, and analgesia needs in addition to improving patient satisfaction during recovery.
Music as an intervention has wide applicability during burn treatment. Particularly as a form of complementary and alternative medicine (CAM), music therapy has been widely used in multiple clinical fields due to its non-pharmacological, non-invasive and easily accessible features. The study of music interventions for burn patients began in the late 1970s. Christenberry published the first paper regarding the application of music therapy for burn patients and outlined a corresponding protocol for the intervention [20].

Music intervention is widely used during dressing changes and debridement to help decrease pain and anxiety in burn patients. The majority of past studies have indicated that music has positive effects with regard to the alleviation of pain for burn patients, especially non-severe pain [4, 21-27]. In addition, Robb et al. [28] found that music assisted relaxation and decreased anxiety in burn patients and increased their compliance during debridement and dressing changes. However, some discrepancies exist regarding the outcomes produced by the clinical application of music therapy for pain management. Ferguson [4] studied the effects of relaxing music on perceived levels of pain and anxiety during range-of-motion exercises and found that the music produced no significant effects on pain relief. Furthermore, there were no significant differences between pretest and post-test anxiety scores following the music intervention. Another study published in 2006 showed that the effects of music interventions on pain and anxiety in pediatric patients during donor-site dressing changes were not conclusive [29]. Thus, the effects of music intervention remain unclear and require further investigation. In previous studies, the primary types of music intervention investigated have included music therapy and music medicine. Music therapy is an interpersonal process during which professional staff who have completed an approved music therapy program use music and all of its facets-physical, emotional, mental, social, aesthetic, and spiritual-to help clients accomplish individualized goals [30]. Music medicine involves relatively passive listening to pre-recorded music offered by a researcher or clinician without the involvement of a music therapist or a defined therapeutic process.

Few reviews have been reported regarding the use of music interventions for burn patients; indeed, only two studies [31, 32] have reviewed the effects of music therapy on burn patients, and these two studies were not metaanalyses. Other studies have reviewed the effects of nonpharmaceutical therapy, which is not restricted to music therapy or music medicine on burn patients [3, 33]. No review study thus far has conducted a meta-analysis examining the effects of music interventions on burn patients. The purpose of the current systematic review and meta-analyses was to evaluate the effects of randomized 
controlled trials (RCTs) of music interventions for burn patients during treatment procedures and to provide recommendations for future research and clinical practice.

\section{Methods}

\section{Search strategy}

The study was designed in accordance with the Cochrane Handbook for Systematic Reviews of Interventions. Our results were reported according to the Preferred Reporting Items for Systematic Reviews and Meta-Analysis statement [34]. We performed a search of all literature regarding the clinical application of music therapy on burn patients using the following databases: MEDLINE (via PubMed), EMBASE, Cochrane Library, Psychinfo, VIP and CNKI. We searched the databases from their earliest available dates through February 2016. Both MESH terms and free text words describing the use of music interventions (including music therapy and music medicine)' and 'the measurement of physical activity outcomes (including pain, anxiety, burn characteristics, dressing changes, wound care, debridement and rehabilitation) were used in the search. The articles of these two sets were then combined using the Boolean 'AND' operator. The search builders were presented as follows: 'music' or 'music intervention' or 'music therapy' or 'music medicine' AND 'burn' or 'burn patient"' or 'burn pain' or 'burn anxiety' or 'dressing changes' or 'debridement' or 'wound care' or 'burn rehabilitation (Additional file 1: Table S1). Reference checking and citation tracking of the included articles were manually performed to identify additional studies meeting the inclusion and exclusion criteria. In addition, we manually searched the Chinese databases of journals, dissertations and magazines for related articles as well as the references to these articles.

\section{Inclusion and exclusion criteria}

The inclusion criteria were RCTs with a parallel group, crossover or cluster design that included burn patients undergoing various procedures (e.g., dressing changes, debridement, range of motion exercises, and surgery). The subjects in the intervention group received music intervention before and/or during and/or after procedures, whereas the subjects in the control group underwent procedures without music. The music interventions included music therapy and music medicine. The music could be live music or recorded music, and the styles of music were not limited. Studies were excluded if their raw data could not be extracted or if music was not the main intervention method used during treatment, such as interventions that involved music combined with massage. Each article should be scored according to the Cochrane Collaboration's tool for assessing risk of bias and those less than 2 points should be excluded. Literature studies written in English and Chinese have been included in this manuscript.

\section{Data extraction}

Primary outcome measurement in this meta-analysis was pain intensity, while anxiety was considered a secondary outcome measurement. Data were carefully and independently extracted from all eligible studies by two investigators (JL, ZL according to the inclusion criteria mentioned above using a prespecified Microsoft Excel spreadsheet. The extracted data included study characteristics (e.g., author name, year of publication, sample size, patient age, total body surface area (TBSA) and type of music), effect measurements (e.g., pain score, level of anxiety, and heart rate), and quality indicators (e.g., adequate sequence generation, allocation concealment, and blinding). Disagreement was resolved by discussion or consulting with a third reviewer (YW).

\section{Risk of bias assessment}

The methodological quality of the studies was independently evaluated by two investigators (JL, ZL) according to the Cochrane Risk of Bias tool for RCTs [35]. Any differences were resolved by consulting with a third reviewer (YW).

\section{Statistical analysis}

Statistical heterogeneity was determined using Q-test and the $I^{2}$ statistic. For cases in which $P \leq 0.10$ and $I^{2} \geq$ $50 \%$, a random effects model was applied. Otherwise, a fixed effects model was used. The endpoints were SMDs and 95\% CIs. Publication bias was assessed using Begg's funnel plot and Egger's test.

\section{Results}

\section{Study selection}

After performing an extensive electronic search combined with a manual search, 491 records were identified, resulting in an initial library of 409 references following the removal of 82 duplicates. 354 records were excluded on the basis of title or abstract. Fifty-five full-text articles reviewed to determine its eligibility for inclusion and exclusion criteria. After an independent review of titles and abstracts, 38 records were excluded for failing to meet the inclusion criteria. A total of 17 RCTs were included in the final review (Fig. 1). The following variables were extracted from the included studies: length of study, size of trial sample, ages and genders of participants, and procedures and intervention methods used. These data are shown in Table 1.

\section{Study characteristics}

The current review included 804 burn patients from 17 RCTs comparing patients undergoing treatments with 


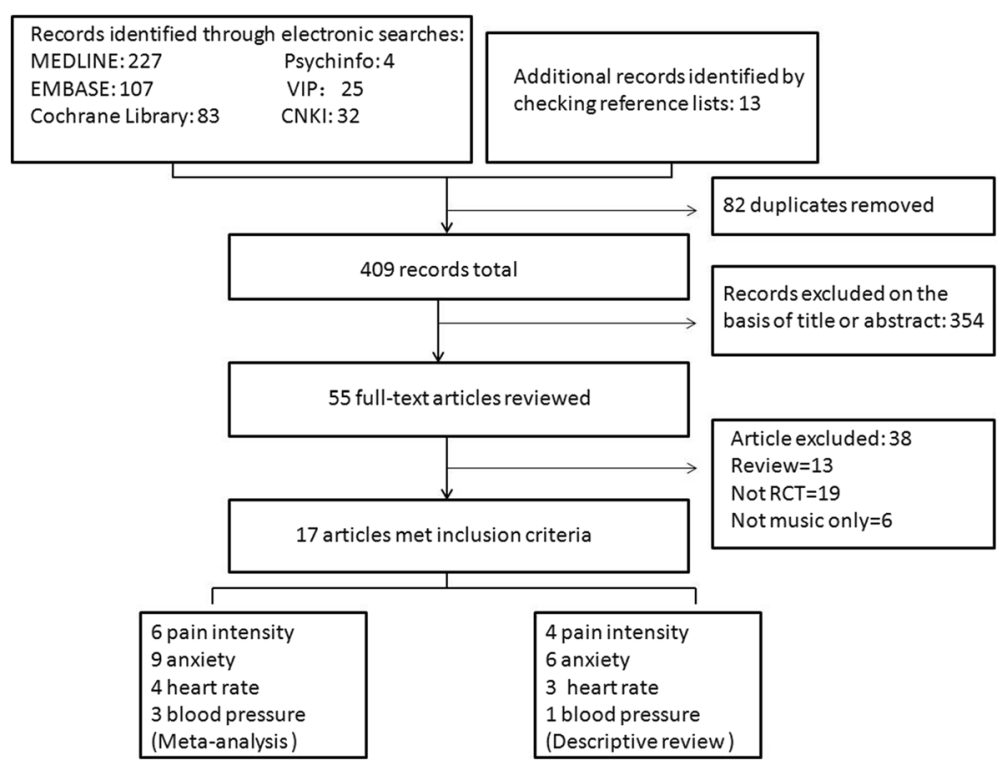

Fig. 1 PRISMA flow diagram: study selection

and without music interventions. Table 1 lists characteristics from all included studies. These trials included nine studies published in Chinese [22, 27, 36-42] and eight studies published in English [4, 24, 25, 28, 29, 43, 44]. The ages of the included patients ranged between 6 and 86 years old. Five studies reported the average age of their patients. From a total of 17 literatures, two literatures with 92 patients didn't provide gender information. There is a total of 722 patients in the rest 15 literatures, among them, $67.6 \%$ were male patients. The types of procedures investigated included dressing change [22, 29, 37, 43, 44], debridement [24, 26, 36, 39], preoperative procedures [28], range of motion rehabilitation [4], cold therapy [23], daily nursing care [40], isolation [42] and hospitalization [25, 37, 38].

Most of the music used in the intervention was selfselected by the patient $[4,28,29,38,42,44]$ or based on a patient's preferences [22, 24-26, 36]. Recorded music was used in 15 studies, and live music was used in three studies [24, 26, 29] (Table 1). The main methods used for music intervention in the included trials were attention distraction methods such as Muralvision or musical alternate engagement (MAE) and relaxation methods such as music-assisted relaxation (MAR) and music-based imagery (MBI) (Table 1).

\section{Outcome measurements}

Pain intensity was assessed in ten studies [4, 22-26, 29, $36,43,44]$ using the following measurement tools: the Visual Analogue Scale (VAS) [4, 22-25, 36], the Wong/ Baker Faces Rating Scale (WBFRS) [26, 29], the McGill Pain Questionnaire (MGPQ) [43], the Nursing Assessment of Pain Index (NAPI) [29] and the 11-point Likert scales (11-LS) [44]. In one study, pain intensity experienced by pediatric patients was assessed using the WBFRS and the NAPI [29] (Table 1). Only six studies were included in this meta-analysis; the remaining four studies were descriptively reviewed because data could not be extracted from them.

Fifteen studies assessed anxiety descriptors [4, 22-26, 28, 29, 37-43] using the following measurement tools: State-Trait Anxiety Inventory forms (STAI) [4, 22, 23, 28, 43], the Self-Rating Anxiety Scale (SAS) [37, 40-42], the Hamilton Anxiety Scale (HAMA) [39], the Fear Thermometer (FT) [29] and the VAS [24-26, 38] (Table 1). However, six studies were excluded due to a lack of raw data; therefore, only nine studies were included in our analysis of anxiety descriptors (Table 1).

Blood pressure was evaluated based on measurements of systolic blood pressure (SBP) and diastolic blood pressure (DBP). Four studies reported the effects of music intervention on SBP and DBP $[22,25,28,41]$; of these, three were included in the meta-analysis [22, 25, 41].

Heart rate, another continuous variable in terms of vital signs, was extracted in four studies and combined in the meta-analysis [22, 25, 29, 41]; of these, three studies provided only descriptive reviews and were not included in the meta-analysis $[26,28,37]$.

\section{Risk of bias}

To assess the risk of bias, the patients were randomly allocated into two groups; however, the majority of included studies did not describe their exact methods of randomization [4, 22-26, 28, 29, 36-39, 43, 44]. Only three studies claimed that allocation was based on the generation of a random number table $[40,41]$ or 


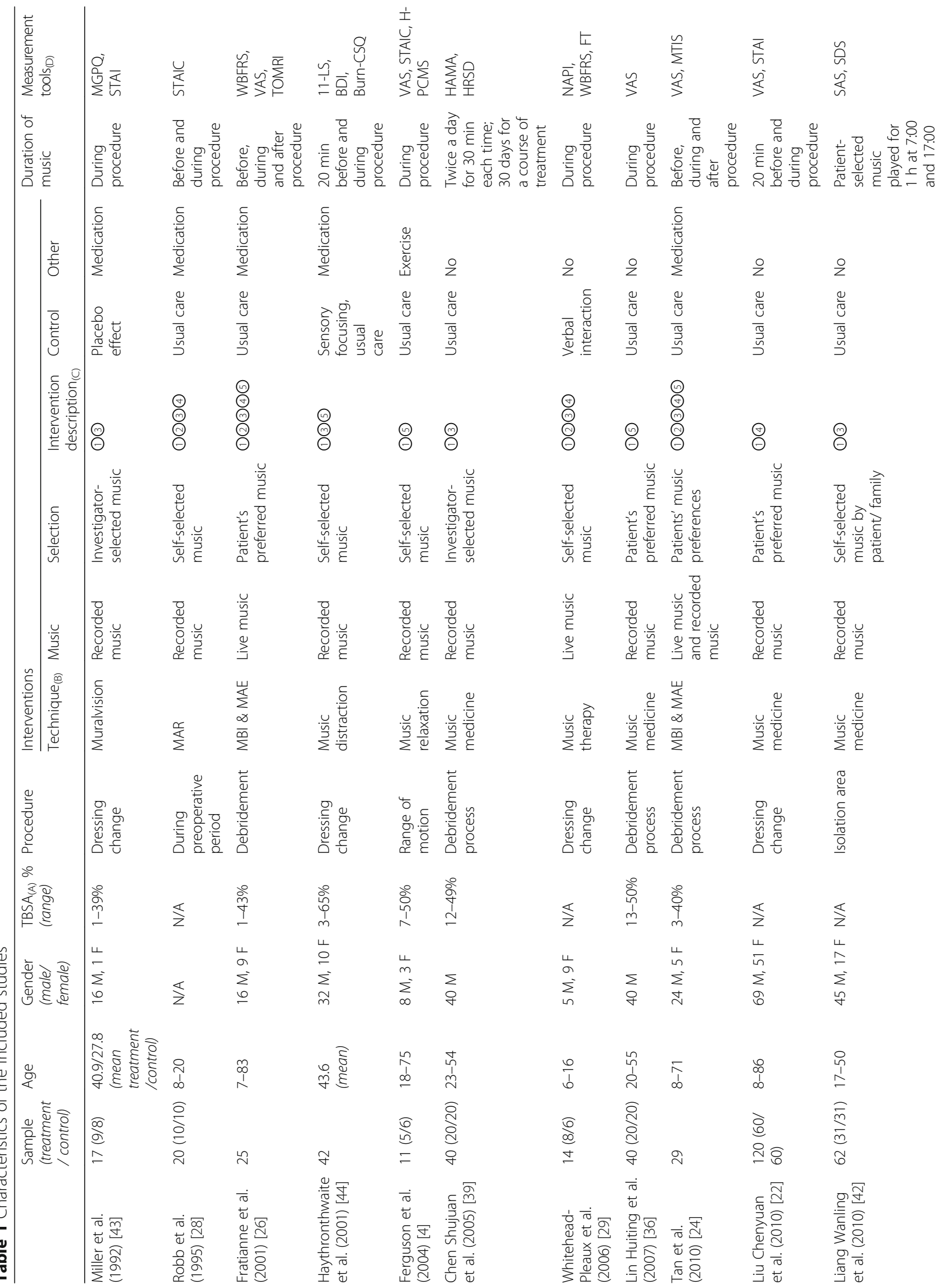




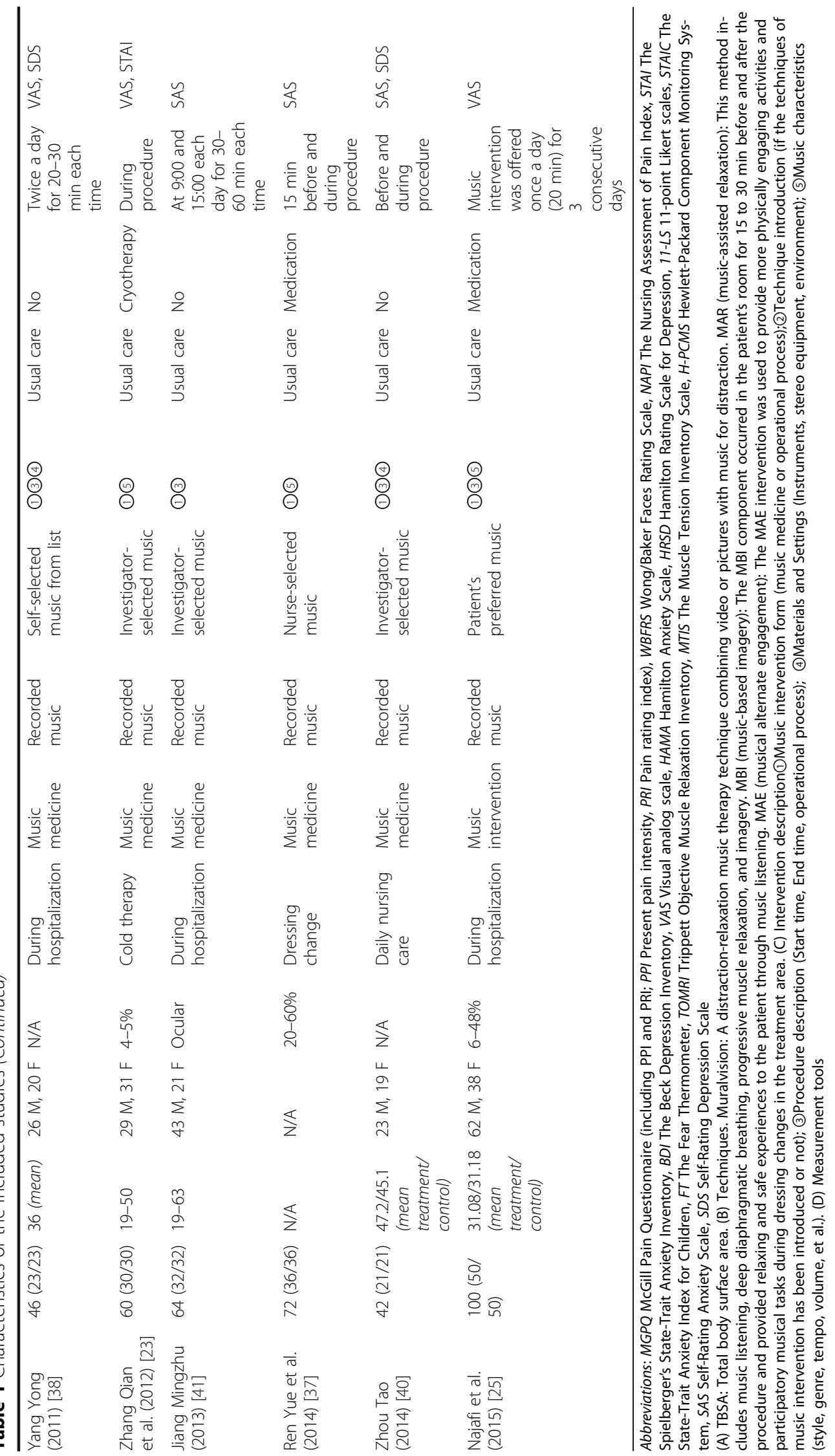


random lottery [42]. The blinding of treatment allocation was obtained by concealed envelopes in one study [24]. Because of the nature of music intervention, the evaluation criteria used for double-blinding were obscure for most studies. Blinded methodology was used as much as possible in the included studies. In one study, the physicians treating the patients were blinded regarding whether the patients were listening to music prior to treatment [26]. In Najafi et al. study, a blinded co-researcher recorded and measured the experimental data [25]. In another study, to decrease rater bias, the research nurses were not assigned to the patients in the research group prior to the study [24]. In addition, two of the studies did not provide any measurement raw data but just final results and therefore demonstrated possible outcome-reporting bias [37, 38] (Fig. 2).

\section{Outcomes of meta-analysis}

\section{Primary Outcome}

Pain. The meta-analysis of six trials and 260 burn patients for measures of pain intensity demonstrated significant heterogeneity $\left(I^{2}=81.6 \%, P<0.001\right)$. The pooled result from the random effects model demonstrated significant differences in pain scores between the music intervention group and the nonmusic intervention group (SMD $=-1.26,95 \% \mathrm{CI}$ $[-1.83,-0.68])[23-25,29,36,43]$ (Fig. 3). Music intervention was found to reduce the pain experienced by burn patients during treatment procedures.

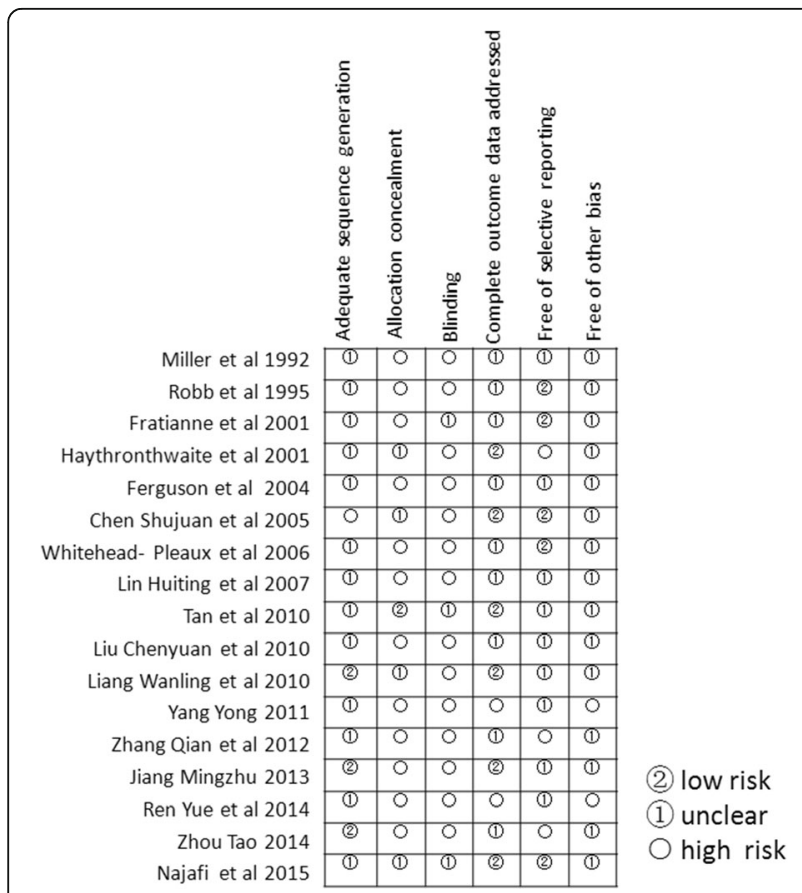

Fig. 2 Results of bias risk assessments
Four studies were included in descriptive reviews. In Fratinanne et al. [26] study, self-reported pain was improved in the music therapy group by over four intervals during treatment procedures. The selfreporting of pain was significantly decreased for those who received music therapy compared to those who did not. Liu Chenyuan et al. [22] study reported that 98.33\% of patients had level 0 or level 1 pain during dressing changes in the experimental group, while only $80 \%$ of patients in the control group had similar low pain levels. The majority of patients in the control group had significantly higher pain levels than those in the experimental group during dressing changes. However, contrary evidence was reported in other studies. Haythronthwaite et al. found that patients in a sensory focusing group experienced greater pain relief than those in a music distraction group based on serial pain ratings [44]. In Ferguson's study, although there was a difference between pretest and post-test pain across groups, no difference in pain was found between the groups [4].

Secondary Outcomes

Anxiety Level. The included anxiety scores demonstrated statistically significant heterogeneity $\left(I^{2}=87.0 \%, P<0.001\right)$. The results showed a statistically significant reduction in the anxiety levels of the burn patients $(\mathrm{SMD}=-1.22,95 \%$ CI $[-1.75$, $-0.69])$ in the intervention group compared to those in the control group [23-26, 29, 36-39, 41-43]

(Fig. 4).

Although the study reported by Robb et al. [28] did not include sufficient data to be included in the meta-analysis, a significant decrease in anxiety scores was found for the experimental group compared to the control group.

Zhoutao reported that music intervention had a significant positive effect on anxiety alleviation; the effective ratio of the control group was $9.52 \%$, whereas the effective ratio of the experimental group was $52.38 \%(P<0.05)$ [40].

Although two studies that were conducted in China were not included in the meta-analysis due to a lack of pretest raw data, the results of these studies also indicated that music interventions significantly reduced anxiety for severe burn patients [38] during hospitalization or during dressing change when combined with anesthetics [37]. Fratinanne et al. [26] indicated that self-reported anxiety during medical procedures was reduced by four intervals in the music therapy group, but no statistical significance was observed. Moreover, Ferguson and Voll also reported that no significant reduction in anxiety was found during therapy including music relaxation [4].

Heart Rate. The effects of music intervention on heart rate during burn treatment procedures were extracted from four studies in the meta-analysis [22, 25, 29, 41], and the statistical heterogeneity for this variable was 


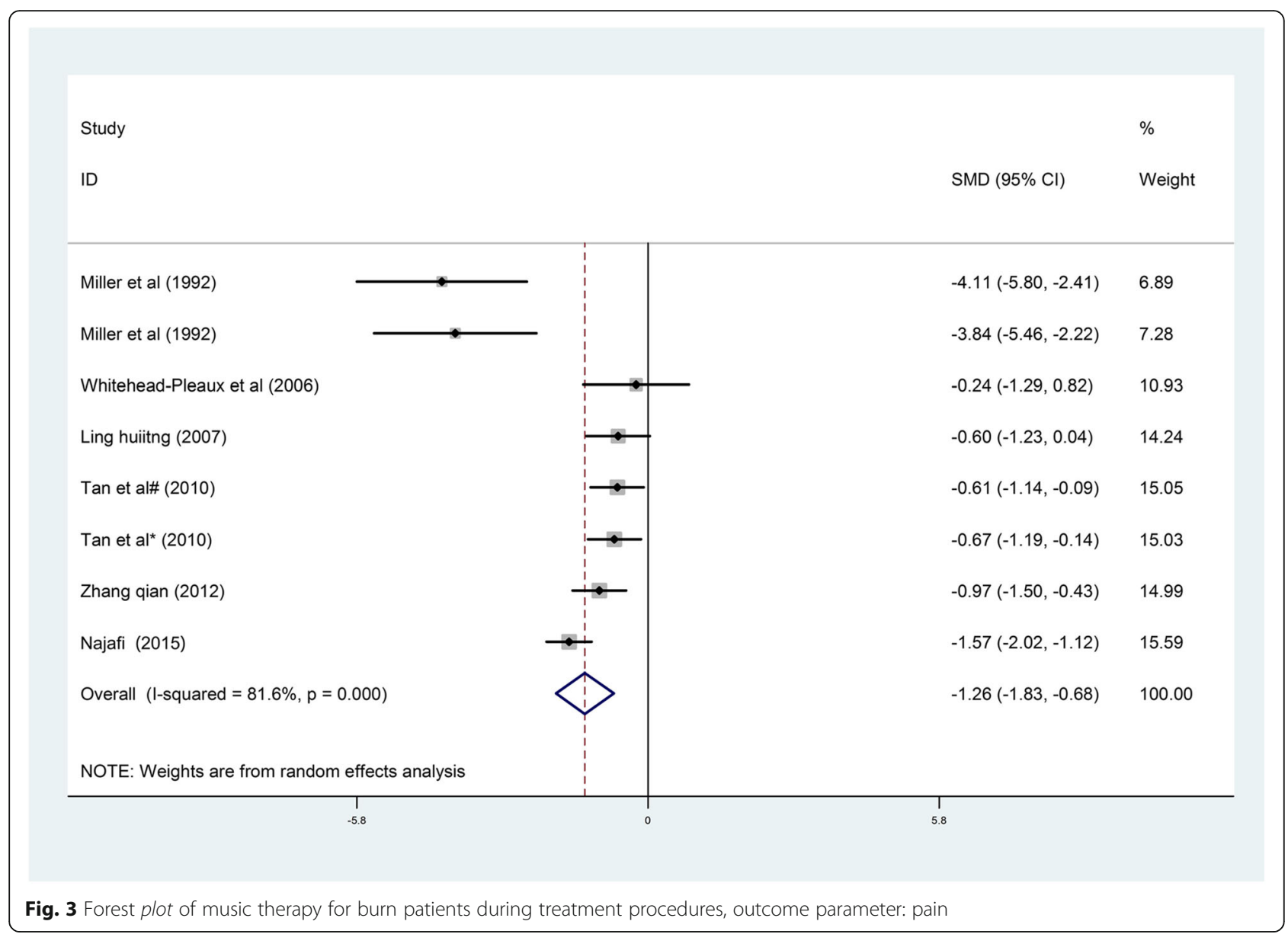

significant $\left(I^{2}=88.8 \%, P<0.001\right)$. Compared with the usual care group, heart rate was significantly decreased in the music intervention group (SMD $=-0.60,95 \% \mathrm{CI}$ $[-0.84,-0.36])$ (Fig. 5).

Three studies that had reported the effects of music interventions on heart rate were not included in the metaanalysis due to ineligibility. Robb et al. [28] indicated that music interventions showed no significant effect on heart rate between pre- and post-test periods for either group. Frantianne et al. [26] reported that music therapy had a slight effect on heart rate, although the difference was not significant. However, in Renyue et al. [37] study, the posttest results revealed that music interventions decreased heart rate significantly during dressing changes compared to the control group.

Blood Pressure. Four studies reported on the effects of music interventions on blood pressure [22, 25, 28, 41]; of these, three were included in the meta-analysis. The random effects pooled result did not demonstrate differences between the intervention group and the control group with regard to blood pressure during treatment procedures (SBP: SMD $=-0.37,95 \% \mathrm{CI}[-1.18$, 0.45]; DBP: SMD $=-0.24,95 \%$ CI $[-0.68,0.20])[22,25$,
41] (Figs. 6 and 7). Similarly, Robb et al. [28] study found no significant differences in heart rate between the preand post-test period for either group.

Respiration Rate. Two of the four studies that included information regarding the effect of music therapy on respiration rate showed statistically significant differences between pre- and posttreatment measurements of respiratory rate across the groups $[4,25]$. The other two studies showed no significant difference in respiration between groups during the preoperative period or during dressing changes $[28,29]$.

\section{Publication bias}

Publication bias was estimated using Begg's test (for pain, $\mathrm{z}=-1.43, P=0.202$; for anxiety, $\mathrm{z}=0.36, P=0.721$ ) and Egger's linear regression test (for pain, $\mathrm{z}=1.11, P$ $=0.266$; for anxiety, $t=-1.18, P=0.271)$. The results suggested that there was no significant evidence of publication bias (Additional file 2: Figure S1). Furthermore, the results of sensitivity analysis indicated that the overall results in the meta-analysis were robust and reliable. 


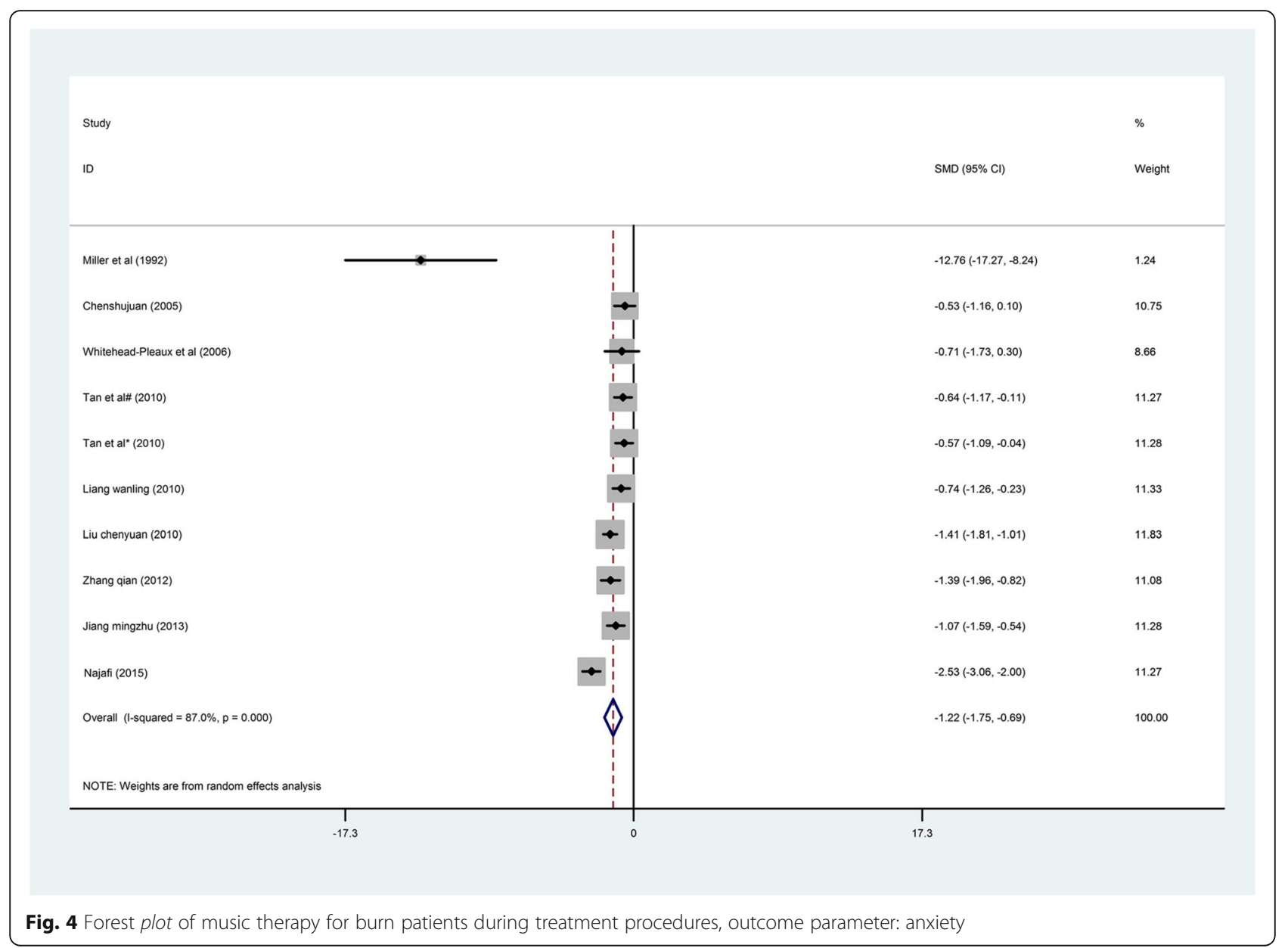

\section{Discussion}

The purpose of the current systematic review was to evaluate the effects of music interventions on burn patients undergoing medical procedures.

During burn treatment and nursing, many factors cause patients to experience pain, including the wound itself, dressing changes, bathing, debridement, excision and grafting. Furthermore, escharotomy, plastic surgery, routine occupational therapy, physical therapy, nursing care and rehabilitation therapy also induce pain. In the current systematic review and meta-analysis, music was used as an intervention in seven different types of procedures, including dressing change, debridement, range of motion exercise, preoperative preparations, cold therapy, nursing care and isolation. The effects of music intervention in other clinical fields, including for critically ill patients receiving mechanical ventilatory support [13] and coma patients [45], has been well studied. These studies provide a reference for the application of music therapy for burn patients in the ICU.

The current meta-analysis showed a significant decline in pain intensity before and after patients received music interventions. The majority of studies showed that music had a positive effect on pain relief; the burn patients exposed to music typically reported low to moderate amounts of pain during treatment. This result is consistent with results from studies of the use of music for the relief of chronic, non-severe pain [46]. However, one of the studies included in the current systematic review and meta-analysis showed that music therapy not only reduced chronic pain but also severe pain [24]. A prospective, randomized crossover clinical trial was conducted in an inpatient burn unit using MBI and MAE. The study indicated that music therapy decreased pain, anxiety, and muscle tension in burn patients during acute procedures [24]. So far, Tan's findings were different than that of Fratianne [26] and Prensner [47]. Therefore, larger sample studies regarding the effect and application field of MBI \& MAE are needed in the future.

It is well known that the most widely accepted neurological principle underlying the mechanism for the association of music and pain relief is the gate control theory of pain reported by Melzack and Wall [24, 29, 48]. This theory asserts that stimulation by non-noxious input is able to suppress pain. However, a recent study reported 


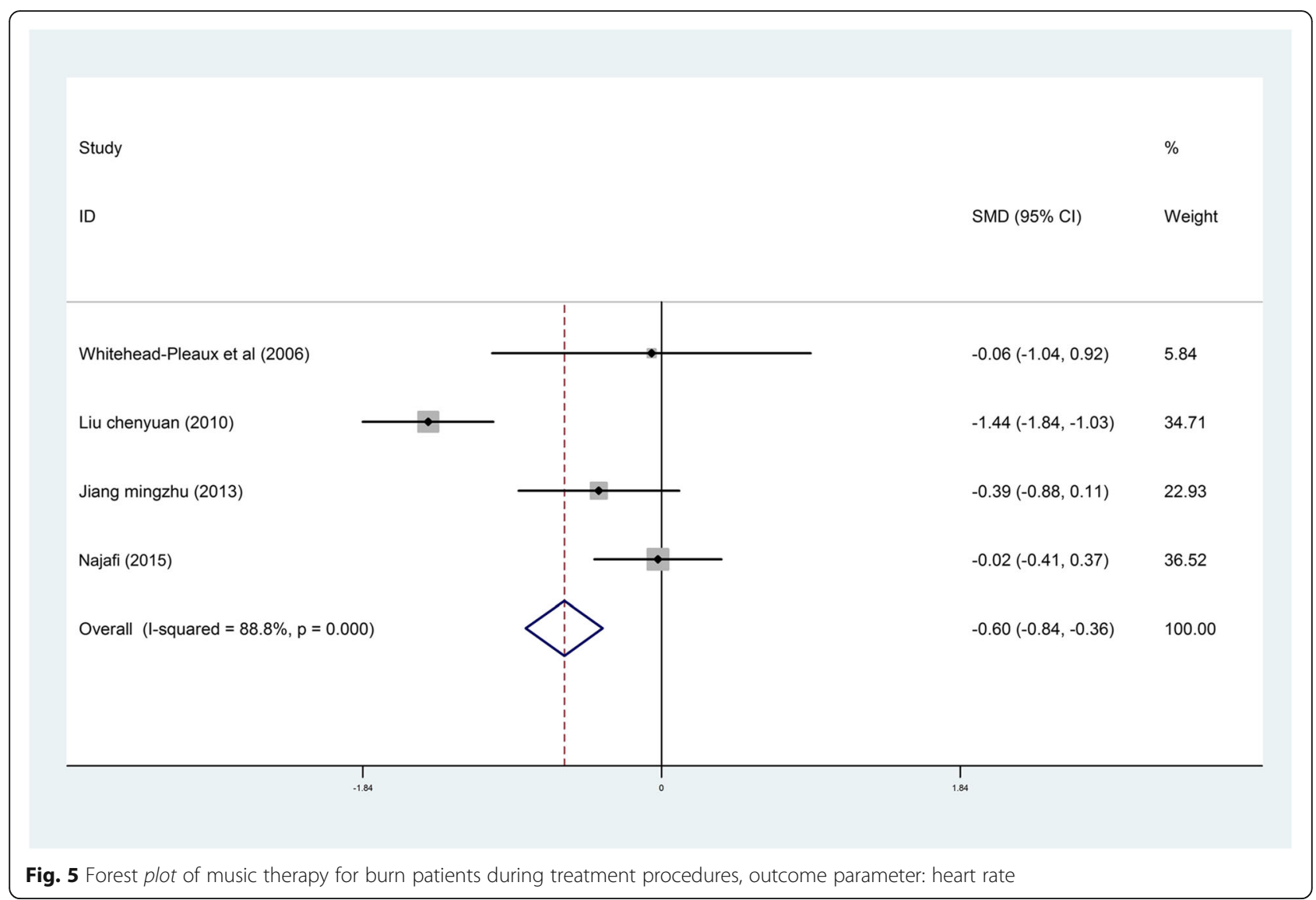

that music therapy modulates pain perception through at least two different mechanisms that involve changes in the activity of delta and gamma bands at different stages of pain processing [49]. These results provided novel insights into the neurological principals that underlie the achievement of pain relief following music therapy. It is necessary to find a solution to resolve the pain and anxiety felt by burn patients undergoing treatment procedures.

The use of music as an intervention has shown the potential to reduce pain during burn treatment. Haythronthwaite's study indicated that the effect of music on pain relief was more obvious in a sensory focusing group compared with a music distraction group [44]. It is worth noting that the music intervention methods used in the music distraction group fell under the purview of medicine rather than music therapy. Thus, if rigorous music therapy methods were introduced into this research according to treatment target, they may produce the same effects as those observed in the sensory focusing group. Tan's study also proved this point [24]. More studies are needed to reveal the roles of music intervention, especially music therapy, in severe pain control. However, based on the above-referenced study by Hauck et al. [49], the combination of relaxation and distraction with music intervention may help patients cope with pain. Although individual studies have shown that submitting burn patients to music interventions provides some evidence of decreased pain intensity and anxiety, there have been no indications that any specific type of music offers more benefits.

Consistent with previous studies, our study believed that it is vital to establish appropriate standard protocols of music therapy during different burn treatment procedures [32]. In this study, four kinds of music therapy protocols including Muralvision, MAR, MBI and MAE have been used in these included studies. One literature which was not included in this Meta-analysis introduced the effect of other music therapy protocols including Song Phrase Cued Response (SPCR)、Adapted Progressive Muscle Relaxation (APMR)、MBI and the Relaxation Response Elicitation (RRE) during burn treatment. Those different protocols have been adapted to meet the specific needs of burn patients during specific procedures [47]. However, further researches are still needed to provide evidencebased clinical practice of music therapy protocol for patients with specific needs.

Furthermore, in most studies, the music was selected by patients from existing music lists or was the patient's own preferred music. However, in Chinese studies, 


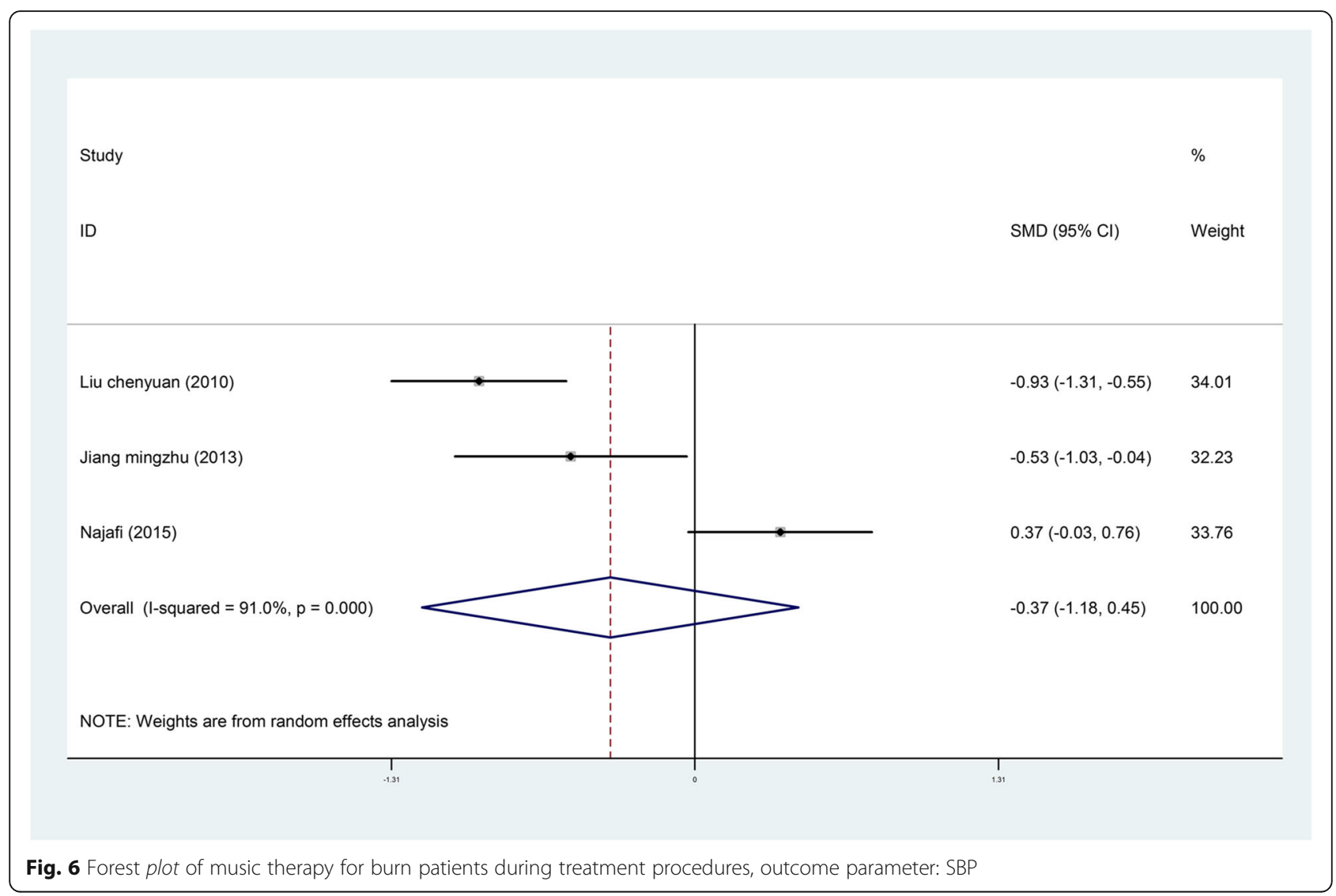

music interventions have mainly relied on music medicine. In China, the quality of music intervention still needs improvement due to the lack of professional music therapists and standardized training. The improvement of professional music therapy may promote future research into music therapy in China.

Eleven studies reported significant anxiety relief between the intervention group and the control group. Correspondingly, patient satisfaction also improved during treatment in three studies [37, 38, 42]. However, the results of these trials differed from those in other studies in the review, and they did not provide enough raw data on specific indicators to support the meta-analysis or answer our email requests for more detailed data [4, 44].

More appropriate measurement scales and methods for relieving pain and anxiety are needed for burn patients. In the 17 studies included here, many different scales were used to measure pain and anxiety. The VAS, the MGPQ, the WBFRS and the NAPI were used to measure pain, while the STAI, the VAS, the FT, the HAMA and the SAS were used to measure anxiety. However, Tan's research showed that the graphic rating scale, the MGPQ and the STAIR are not suitable for the study of burn patients since these measurements are all subjective self-reports. However, this point of view is still controversial Furthermore, Whitehead-Pleaux et al. [29] found that the FT could not capture the effects of music on pain and anxiety on pediatric patients undergoing painful procedures due to their limited understanding of the terms.

Meanwhile, we found that the application of analgesic during burn treatment has received more and more attentions [24, 26, 47]. One study demonstrated a positive correlation between burn patient increased comfort levels when music therapy was used in conjunction with pharmaceutical treatments [32]. However, no data could be extracted from the included literatures regarding the effect of music therapy on analgesic use. Thus, further studies are needed to investigate the effect of music therapy on pain medication during burn treatment. In addition, burn patients not only faced physical pain, but also psychological distress. Therefore, to establish a physical-psychological intervention program becomes necessary for burn patients. The current systematic review and meta-analysis is the first to assess the effectiveness of music interventions on burn patients undergoing treatment. However, the study results should be interpreted in light of its limitations, most of which are related to the original trials. First, in the majority of the 17 included studies, the risk of bias was moderate. The overall trial quality was reduced due to the lack of concealed allocation or blinded therapists assessing outcome 


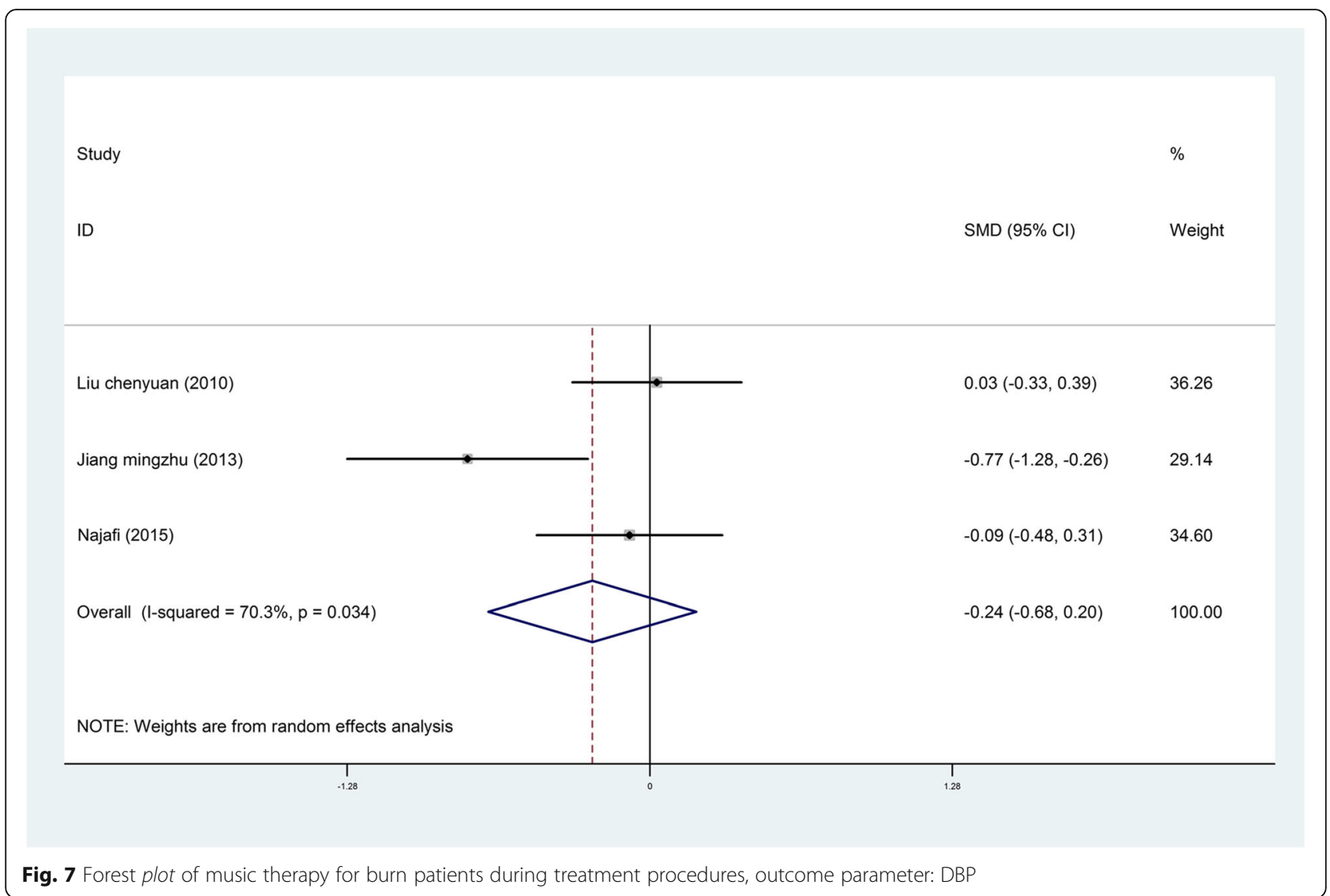

measures. Second, the sample sizes in most of the trials were small. Third, there was heterogeneity in the types of patient populations studied, types of music interventions applied, and types of treatment used. Although heterogeneity existed among the studies, the standardized mean differences per group were calculated, and the results for the pain and anxiety intensity outcomes were pooled. In addition, we would have attempted to adjust for the heterogeneity by performing a subgroup analysis or a meta-regression analysis, but the number of studies was insufficient to perform these analyses. Moreover, some of the studies lacked quantitative measurements of specific indicators, making their inclusion in the meta-analysis risky. However, we included these study results in the review to avoid potential bias.

\section{Conclusions}

In conclusion, our study presents limited evidence from 17 individual trials that burn patients may experience cumulative benefits from music interventions in terms of decreased pain and anxiety, leading to better treatment prognosis. Music intervention has a positive effect on pain alleviation, anxiety reduction and heart rate control, which provides evidence to support the advantages of its use during burn treatment. Further high-quality studies with carefully considered music interventions are recommended to provide more solid evidence on both the short-term and long-term effects of this intervention strategy on burn patients.

\section{Additional files}

Additional file 1: Table S1. Search strategy. (DOC $31 \mathrm{~kb}$ )

Additional file 2: Figure S1. Begg's funnel plot and Egger's linear regression test. (A) Begg's funnel plot for pain. (B) Begg's funnel plot for anxiety. (C) Egger's linear regression test for pain. (D) Egger's linear regression test for anxiety. (JPG $237 \mathrm{~kb}$ )

\section{Abbreviations}

11-LS: 11-point Likert scales; CAM: Complementary and alternative medicine; Cls: Confidence intervals; CNKI: Chinese National Knowledge Infrastructure; CqVip: Chongqing Vip of Chinese Science and Technology Periodical Database; DBP: Diastolic blood pressure; FT: Fear Thermometer; HAMA: Hamilton Anxiety Scale; P': I-square; MAE: Musical alternate engagement; MAR: Music-assisted relaxation; MBI: Music-based imagery; MGPQ: McGill Pain Questionnaire; NAPI: Nursing Assessment of Pain Index; RCTs: Randomized controlled trials; SAS: Self-Rating Anxiety Scale; SBP: Systolic blood pressure; SMDs: Standardized mean differences; STAI: State-Trait Anxiety Inventory forms; TBSA: Total body surface area; VAS: Visual Analogue Scale; WBFRS: Wong/Baker Faces Rating Scale

\section{Acknowledgments}

We are grateful to the contribution of Dr. Jun Wu from burn center of southwest hospital for providing helpful advice and comments on the quality appraisal of the included studies. 


\section{Funding}

This research was supported by 2014 Chongqing Youth Social Science Projects, Grant Reference Number: 2014QNYS35.

\section{Availability of data and materials}

All the data and materials which supporting this manuscript are authentic. We would like to share all these data to readers of your journal.

\section{Authors' contributions}

$Y G W$ supervised $J Y L$ and $L Z$ to perform this review and revised the manuscript. JY $L$ and $L Z$ conducted the database search, assessed studies for inclusion, extracted and analyzed the data, and drafted the manuscript. YG W assessed studies for inclusion, extracted the data which followed by cross checking with JY L. L Z was responsible for the statistics analysis. JY L amended English writing of this review. YG W and JY L arbitrated any disagreements. All authors have read and approved the final version of the manuscript.

\section{Competing interests}

The authors declare that they have no competing interests.

\section{Consent for publication}

\section{Not applicable.}

\section{Ethics approval and consent to participate}

Not applicable. All analyses were based on previous published studies, thus no ethical approval and patient consent are required.

\section{Publisher's Note}

Springer Nature remains neutral with regard to jurisdictional claims in published maps and institutional affiliations.

\section{Author details}

'Department of Humanities and Social Sciences, The Third Military Medical University, Chongqing, China. ${ }^{2}$ Research Institute of Field Surgery, Daping Hospital, The Third Military Medical University, Chongqing, China. ${ }^{3}$ The Third Military Medical University, Chongqing 400038, China.

\section{Received: 4 June 2016 Accepted: 7 March 2017}

\section{Published online: 17 March 2017}

\section{References}

1. Lorente JA, Amaya-Villar R. Update in the management of critically ill burned patients. Med Intensiva. 2016:40(1):46-8.

2. de Jong AE, Middelkoop E, Faber AW, Van Loey NE. Non-pharmacological nursing interventions for procedural pain relief in adults with burns: a systematic literature review. Burns. 2007;33(7):811-27.

3. Summer GJ, Puntillo KA, Miaskowski C, Green PG, Levine JD. Burn injury pain: the continuing challenge. J Pain. 2007:8(7):533-48.

4. Ferguson $\mathrm{SL}$, Voll KV. Burn pain and anxiety: the use of music relaxation during rehabilitation. J Burn Care Rehabil. 2004;25(1):8-14.

5. Robert R, Blakeney P, Villarreal C, Meyer 3rd WJ. Anxiety: current practices in assessment and treatment of anxiety of burn patients. Burns. 2000;26(6):549-52.

6. Carrougher GJ, Ptacek JT, Honari S, Schmidt AE, Tininenko JR, Gibran NS, et al. Self-reports of anxiety in burn-injured hospitalized adults during routine wound care. J Burn Care Res. 2006;27(5):676-81.

7. Mejia-Rubalcava C, Alanis-Tavira J, Mendieta-Zeron H, Sanchez-Perez L. Changes induced by music therapy to physiologic parameters in patients with dental anxiety. Complement Ther Clin Pract. 2015;21(4):282-6.

8. Bradt J, Dileo C, Shim M. Music interventions for preoperative anxiety. Cochrane Database Syst Rev. 2013;6:D6908.

9. Engwall M, Duppils GS. Music as a nursing intervention for postoperative pain: a systematic review. J Perianesth Nurs. 2009;24(6):370-83.

10. Lesiuk T. The effect of mindfulness-based music therapy on attention and mood in women receiving adjuvant chemotherapy for breast cancer: a pilot study. Oncol Nurs Forum. 2015;42(3):276-82.

11. Chen X, Seth RK, Rao VS, Huang JJ, Adelman RA. Effects of music therapy on intravitreal injections: a randomized clinical trial. J Ocul Pharmacol Ther. 2012;28(4):414-9.

12. Simavli S, Gumus I, Kaygusuz I, Yildirim M, Usluogullari B, Kafali H. Effect of music on labor pain relief, anxiety level and postpartum analgesic requirement: a randomized controlled clinical trial. Gynecol Obstet Invest. 2014;78(4):244-50

13. Chlan LL, Weinert CR, Heiderscheit A, Tracy MF, Skaar DJ, Guttormson JL, et al. Effects of patient-directed music intervention on anxiety and sedative exposure in critically ill patients receiving mechanical ventilatory support: a randomized clinical trial. JAMA. 2013;309(22):2335-44.

14. Chlan L. Effectiveness of a music therapy intervention on relaxation and anxiety for patients receiving ventilatory assistance. Heart Lung J Crit Care 1998;27(3):169-76.

15. Lin H, Ji PL, Sit JWH, Chung L, Zuo YJ, Wei GM. Effects of music intervention on physiological stress response and anxiety level of mechanically ventilated patients in China: a randomised controlled trial. J Clin Nurs. 2010;19(7-8):978-87.

16. Twiss E, Seaver J, Mccaffrey R. The effect of music listening on older adults undergoing cardiovascular surgery. Nurs Crit Care. 2006;11(5):224-31.

17. Wong HLC, Lopez-Nahas V, Molassiotis A. Effects of music therapy on anxiety in ventilator-dependent patients. Heart Lung J Acute Crit Care. 2001;30(5):376-87.

18. Wang MC, Zhang LY, Zhang YL, Zhang YW, Xu XD, Zhang YC. Effect of music in endoscopy procedures: systematic review and meta-analysis of randomized controlled trials. Pain Med. 2014:15(10):1786-94.

19. Hole J, Hirsch M, Ball E, Meads C. Music as an aid for postoperative recovery in adults: a systematic review and meta-analysis. Lancet. 2015; 386(10004):1659-71.

20. Christenberry EB. The use of music therapy with burn patients. J Music Ther. 1979;16(3):138-48

21. Whitehead-Pleaux AM, Zebrowski N, Baryza MJ, Sheridan RL. Exploring the effects of music therapy on pediatric pain: phase 1. Music Ther. 2007;44(3):217-41.

22. Liu CY, Yuan QF, Zou F. The effect of music therapy on pain and anxiety control for burn patient during dressing change. Chin Cosmet Med. 2010; 19(11):1712-3 [Chinese].

23. Zhang Q, Luan YM, Zou ZQ. A combination of music therapy and cold therapy on pain and anxiety control for upper limbs burn patients during early stage. Chin Rehabil. 2012;27(6):456-7 [Chinese]

24. Tan X, Yowler CJ, Super DM, Fratianne RB. The efficacy of music therapy protocols for decreasing pain, anxiety, and muscle tension levels during burn dressing changes: a prospective randomized crossover trial. J Burn Care Res. 2010;31(4):590-7.

25. Najafi GT, Mohades AF, Rafii F, et al. The Effects of Music Intervention on Background Pain and Anxiety in Burn Patients: Randomized Controlled Clinical Trial.[J]. J Burn Care Res, 2015;37(4):226-34.

26. Fratianne RB, Prensner JD, Huston MJ, Super DM, Yowler CJ, Standley JM. The effect of music-based imagery and musical alternate engagement on the burn debridement process. J Burn Care Rehabil. 2001;22(1):47-53.

27. Chen $X L$, Xie XX, Wu LP, Yang FX, Xie XY. The effect of music interventions on pain for extensive burn patients during dressing change. Chin J Rehabil Theory Pract. 2005;11(10):857-8 [In Chinese].

28. Robb SL, Nichols RJ, Rutan RL, Bishop BL, Parker JC. The effects of music assisted relaxation on preoperative anxiety. J Music Ther. 1995;32(1):2-21.

29. Whitehead-Pleaux AM, Baryza MJ, Sheridan RL. The effects of music therapy on pediatric patients' pain and anxiety during donor site dressing change. J Music Ther. 2006:43(2):136-53.

30. Davis WB, Gfeller KE, Thaut MH. An introduction to music therapy: theory and practice. Silver Spring: American Music Therapy Association; 2008. p. 5-15.

31. Protacio J. Patient-directed music therapy as an adjunct during burn wound care. Crit Care Nurse. 2010:30(2):74-6.

32. Bingham B. The Effect of Music Therapy on Burn Patients[C]. Thesis for: BSN, Advisor: Ms. Joanne Martin, 2014;1-36.

33. Hanson MD, Gauld M, Wathen CN, MacMillan HL. Nonpharmacological interventions for acute wound care distress in pediatric patients with burn injury: a systematic review. J Burn Care Res. 2008;29(5):730-41.

34. Moher D, Liberati A, Tetzlaff J, Altman DG, PRISMA Group. Preferred reporting items for systematic reviews and meta-analyses: the PRISMA statement. PLoS Medicine. 2009;6(7):e1000097. doi:10.1371/journal.pmed. 1000097.

35. Higgins JP, Altman DG, Gøtzsche PC, et al. The Cochrane Collaboration's too for assessing risk of bias in randomised trials. BMJ. 2011;343(2):d5928. 7.

36. Lin HT, Chen YT. The application of music therapy on 20 burn patients. J Fujian Med. 2007:9(5):157-8 [In Chinese]. 
37. Ren Y, Liu JJ, Yin J, Sun JH, Liu LH, Wang HT. The clinical effect of background music used composite anesthesia of burn dressing change. Chin J Clin Ration Drug Use. 2014;7(4):110-1 [In Chinese].

38. Yong $Y$. The effect of music therapy on anxiety and depression alleviation to burn patients. Mod Med Health. 2011;27(6):909-10 [In Chinese].

39. Chen SJ, Xiao XL, Zhang L. The effect of music therapy on anxiety or depression status of severe male burn patients. J Chin Metallurgical Ind Med. 2005;3:328-9 [In Chinese].

40. Zhou T. The effect of music therapy on anxiety relieving for severe burn patients. J Jiling Med. 2014;36:8131-2 [In Chinese].

41. Jiang MZ. The impact of music therapy to physical and psychological status of chemical eyes burn patients. Mod Hosp. 2013;13(9):90-2 [In Chinese].

42. Liang WL, Zhou X, Wen SH, Li YX, Xie YH, Mo MX. The impact of music therapy to severe burn patients' psychological health. Nurs Pract Res. 2010; 07(14):11-3 [In Chinese].

43. Miller AC, Hickman LC, Lemasters GK. A distraction technique for control of burn pain. J Burn Care Rehabil. 1992;5(13):576-80.

44. Haythronthwaite JA, Lawrence JW, Fauerbach JA. Brief cognitive interventions for burn pain. Ann Behav Med. 2001;23(1):42-9.

45. Sun J, Chen W. Music therapy for coma patients: preliminary results. Eur Rev Med Pharmacol Sci. 2015;19(7):1209-18.

46. Standley JM. Music research in medical/dental treatment: meta-analysis and clinical applications. J Music Ther. 1986;23(2):56-122.

47. Prensner JD, Yowler CJ, Smith LF, et al. Music therapy for assistance with pain and anxiety management in burn treatment []]. J Burn Care Res. 2000;22(1):83-8.

48. Melzack R, Wall PD. Pain mechanisms: a new theory [J]. Science. 1967; 150(150):971-9.

49. Hauck M, Metzner S, Rohlffs F, Lorenz J, Engel AK. The influence of music and music therapy on pain-induced neuronal oscillations measured by magnetencephalography. Pain. 2013;154(4):539-47.

\section{Submit your next manuscript to BioMed Central and we will help you at every step:}

- We accept pre-submission inquiries

- Our selector tool helps you to find the most relevant journal

- We provide round the clock customer support

- Convenient online submission

- Thorough peer review

- Inclusion in PubMed and all major indexing services

- Maximum visibility for your research

Submit your manuscript at www.biomedcentral.com/submit
Biomed Central 